\title{
Devenir sujet: les expériences des jeunes sous Contrat jeune majeur en France
}

\author{
Fernanda Cruz Rifiotis \\ Universidade Federal do Rio Grande do Sul, Programa de Pós-graduação em Antropologia Social, \\ Porto Alegre/RS, Brasil
}

\section{Résumé}

Il s'agit des résultats d'une recherche ethnographique menée entre 2015 et 2017 à propos des modes par lesquels les " jeunes majeurs " d'une association éducative parisienne se construisent en tant que sujets. Dans un contexte de contractualisation des politiques de protection françaises, je me suis intéressée aux expériences des jeunes de cette association vis-à-vis du Contrat jeune majeur (CJM), qui est une technologie de gouvernement pour faire face aux effets de la réduction de la majorité depuis 1974, ainsi qu'aux lacunes qu'elle a créé en matière de protection et d'assistance aux jeunes entre 18 et 21 ans qui sont en difficultés d'intégration sociale. L'article fait état d'un chevauchement des questions économiques sur celles d'ordre éducatif de telle sorte que les jeunes qui ont ce contrat ne sont pas ceux qui se trouvent en difficultés d'intégration, mais ceux qui ont un " projet de vie consistant".

Mots clés: Technologie de gouvernement; Contrat jeune majeur; Jeunes; Sujet. 


\section{Sujeitos em devir: as experiências de jovens em situação de "Contrat jeune majeur" na França}

\section{Resumo}

Trata-se de resultados de uma pesquisa etnográfica empreendida entre 2015 e 2017 sobre os modos pelos quais os "jeunes majeurs" (jovens de 18 a 21 anos) de uma associação educativa parisiense se constroem enquanto sujeitos. Em um contexto de contratualização das políticas de proteção francesas, eu me concentrei nas experiências dos jovens dessa associação em relação ao Contrat jeune majeur (contrato jovem adulto), que é uma tecnologia de governo implementada para enfrentar os efeitos da redução da maioridade implementada desde 1974, como também as lacunas que essa criou em termos da proteção e da assistência aos jovens entre 18 e 21 anos que enfrentam dificuldades de integração social. $O$ artigo problematiza a sobreposição das questões econômicas sobre aquelas de ordem educativas, de tal modo que os jovens que têm a oportunidade de assinar o contrato não são exatamente aqueles que encontram dificuldades de integração, mas sim aqueles que possuem um "projeto de vida consistente".

Palavras-chave: Tecnologias de governo; Contrato; Jovens; Sujeito. 


\title{
Devenir sujet : les expériences des jeunes sous Contrat jeune majeur en France
}

\author{
Fernanda Cruz Rifiotis
}

\section{Introduction}

Dans cet article, j'analyse les façons par lesquelles les jeunes majeurs peuvent se construire en tant que sujets (les modes de subjectivation), compte tenu des logiques de contractualisation qui ont marqué les politiques de protection françaises. Par conséquent, je prends comme objet central de ces politiques le Contrat jeune majeur (CJM), qui sera problématisé comme une technologie de gouvernement. La réflexion est basée sur l'ethnographie que j'ai développée entre 2015 et 2017, dans le cadre de mon postdoctorat en Anthropologie sociale à l'EHESS de Paris. La recherche a comme outil privilégié l'écoute des récits des expériences des jeunes majeurs (à partir d'entretiens et de conversations informelles) et l'observation de leur vie quotidienne au sein de la Suite éducative ${ }^{1} d^{\prime}$ une association parisienne.

Dans le cas des jeunes « sortant du dispositif de protection de l'enfance »" la question de leur " devenir » est centrale pour les politiques publiques et pour les études scientifiques. "Qui sont ces jeunes ?», "Que font-ils ?», "Qu'est-ce qui leur est arrivé après leur départ ?», ces questions sont souvent soulevées par les professionnels qui travaillent dans le dispositif de protection de l'enfance, ainsi que par des chercheurs de différents domaines (Sociologie, Démographie, Psychologie, Droit, etc.). L'avenir de ces jeunes semble éveiller l'attention de tous les intervenants sociaux, en particulier ceux qui participent à la création et à la mise en œuvre des politiques de protection. Dans la perspective plus traditionnelle, il y aurait l'idée d'un investissement pour ces jeunes, qui devrait être rétribué selon ce que sera leur avenir : l'insertion professionnelle, l'achèvement des études, la conquête de l'autonomie, etc. Et quand ces jeunes ne correspondent pas à cet idéal des politiques publiques, ils sont considérés comme ceux qui n'ont pas atteint un « bon projet de vie ».

La recherche que je mène depuis 2010 (Cruz, 2014) est basée sur une espèce d'inversion temporelle dans l'ordre de ces questions, afin de considérer non pas ce que ces jeunes sont devenus (en tant que produit des politiques de protection et de l'institutionnalisation), mais ce qu'ils peuvent devenir en tant que sujet. Dans mon approche analytique, le mot « devenir » a une portée philosophique, une catégorie analytique et, au-delà du sens de changement comme processus d'arrivée à un point, comme identité figée, il prend en considération la construction permanente sans un aboutissement et sans une direction univoque. Cette perspective permet de réfléchir sur les manières dont les jeunes en situation de contrat se mobilisent pour résister et s'adapter à ce qui leur est demandé.

\footnotetext{
1 La Suite éducative est située dans le zoe arrondissement, le deuxième plus peuplé de Paris. Le quartier est connu pour accueillir les populations des migrants et les principales communautés présentes proviennent du Maghreb et de l’Asie (principalement de Chine). La réalité du quartier est comme celle de la Suite. Le service accueille, en moyenne, 40 jeunes âgés de 18 à 21 ans, de différentes provenances géographiques, mais principalement de pays africains comme la Guinée, le Congo et la Côte-d'Ivoire. La Suite éducative est l'endroit où est fait le travail du suivi éducatif des jeunes. C'est dans la Suite que les jeunes sont suivis par une équipe de quatre éducateurs, une chef de service et un psychologue. Chaque jeune a un «éducateur de référence " qui est chargé de son orientation pour qu'il puisse accomplir le projet établi dans le Contrat jeune majeur.

2 Selon la revue de littérature publiée par l'Observatoire de l'enfance en danger (ONPE), en octobre 2014, l'expression " jeunes sortant du dispositif ", qui a pour équivalent l'expression anglaise « care-leavers ", " permet de ne pas fixer un seuil d'âge, mais de s’intéresser plus généralement à la période qui suit le départ du jeune de la dernière structure au sein de laquelle il a été accueilli au titre de la protection de l'enfance ». ONPE (Observatoire national de la protection de l'enfance). Revue de Littérature : L'accompagnement vers l'autonomie des jeunes sortant du dispositif de protection de l'enfance, 2014. Disponible sur Internet : http://www.laurent-mucchielli.org/
} 
Ma proposition est de mettre en avant-plan de mon analyse le sujet d'une façon que « nous ne sommes pas habitués à voir ", c'est-à-dire celui qui s'est construit de façon contingente à partir de ses multiples expériences (Strathern, 2006). Pour une telle entreprise, il est essentiel d'observer les expériences des jeunes majeurs afin de comprendre les modes de subjectivation qui sont révélés par une " attitude générale " (leurs manières d'être dans le monde, d'agir, d'être en relation avec l'autre, d'une certaine manière de faire face aux choses) des jeunes par rapport à eux-mêmes, aux autres et au monde (Foucault, 2010, p.11) (c'est moi qui traduit). Sans perdre de vue que chacun, " avec son nom, son individualité désigne une multiplicité " (Deleuze, Guattari, 1997, p.36) (c'est moi qui traduit). Contrairement à ce que l'on pourrait vouloir, il ne s'agit pas de dire qui sont ces jeunes (et leur devenir) et de les définir à partir de leurs expériences. Ils sont en devenir (dans le sens philosophique), et donc ils échappent à toute prétention de les encapsuler en catégories, en particulier celles qui portent le supposé poids ou les effets de l'institutionnalisation. Ce que je veux présenter est peut-être une partie du " devenir ", une partie de ce qu'ils peuvent en tant que sujets et non de ce qu'ils sont : dans ce sens, il n'y a pas de place pour la substance. Dire ce qu'ils peuvent révèle la dimension de puissance contenue dans chacun de ces jeunes, une multitude de croisements de différentes forces et intensités qui ne convergent pas à une forme finie.

\section{Le Contrat Jeune Majeur en tant que technologie de gouvernement}

Prendre les technologies de gouvernement comme objet d'analyse, cela signifie, d'abord, comprendre que celles-ci sont intégrées dans un problème plus vaste nommé par Foucault (2008) "gouvernementalité ". Le concept qui émerge d'une manière localisée, se référant au système de pouvoir mis en place au XVIIIe siècle (comportant comme principale cible - la population -, comme principal moyen de savoir - l'économie politique - et comme instruments techniques - les dispositifs de sécurité), prend peu à peu un sens plus général et abstrait. En ces termes, il ne définit pas seulement une relation de pouvoir, mais un domaine spécifique lié aux techniques de gouvernement « sous-jacent à la formation de l'État moderne ». Le concept permet de problématiser une sorte de " surévaluation du problème de l'État » ou " le monstre froid face aux individus ", ou encore sous sa forme réduite à un certain nombre de fonctions présentes dans le cours de l'Histoire. Vu que l'État n’a pas « cette unité, cette individualité, cette fonctionnalité rigoureuse », il faut capturer son mode de gouvernement à partir d'un ensemble de techniques et de pratiques hétérogènes (Foucault 2008).

Considérant que l'État est une "réalité composite ", qui sera composée d'une série de fragments de pratiques et de connaissances qui, lorsqu'ils sont assemblés, ne constituent pas une nouvelle forme, car toujours inachevée, les technologies de gouvernement sont devenues « question politique fondamentale et l'espace réel de la lutte politique " (Foucault 1979). Conformément à ce point de vue, les technologies de gouvernement peuvent être considérées comme l'ensemble multiple de stratégies et de tactiques, de connaissances, de dispositifs, de procédures et de réflexions qui permettent de gouverner et objectiver les sujets, les pratiques sociales et les moralités.

Les technologies de gouvernement ne sont pas des « outils neutres », elles sont ainsi inextricablement liées non seulement aux " formes de résistance » et aux « contre-conduites », dans les termes de Foucault ${ }^{3}$, mais aussi à la création des conditions pour l'émergence des formes de se construire en tant que sujet, celles-ci n'étant pas des formes finies, mais toujours en train de se faire et dans une relation expérimentale avec elles-mêmes (Schuch, Ribeiro, Fonseca, 2013). Les technologies de gouvernement, dans cette perspective, sont indissociables

\footnotetext{
3 En suivant cette perspective, il me semble que le dialogue avec Foucault est productif, en particulier en ce qui concerne la notion de modes de subjectivation. Les modes de subjectivation ont peu à voir avec le sujet ou le retour à celui-ci : ils ne correspondent pas à une substance. " (Le sujet) C'est une forme, et cette forme n'est pas surtout ni toujours identique à elle-même. Il y a sans doute des rapports et des interférences entre ces différentes formes du sujet, mais on n'est pas en présence du même type de sujet " (Foucault, 2012, p.269). Les modes de subjectivation peuvent être pris comme forme, parce qu'ils sont variables, selon le temps et les conditions de leur émergence, ils peuvent à tout moment être défaits et refaits.
} 
des modes de subjectivation. C'est-à-dire qu'il ne s'agit pas d'une simple approche par les sujets ni de leur autonomie et ses marges de manœuvre face aux technologies de gouvernement. Elles révèlent l'émergence des sujets selon leurs façons d'évaluer les codes et les institutions, et ainsi de créer des modes d'existence.

Les technologies de gouvernement sont des éléments actifs dans la construction de nouveaux contextes politiques et elles sont toujours remodelées et actualisées (Schuch, Ribeiro, Fonseca 2013). Loin d'un fonctionnement selon des modalités univoques et à partir de mécanismes verticaux, elles procèdent à partir de logiques multiples et de manière diffuse (Fassin, Memmi, 2004). Dans le cas des technologies de gouvernement envers les jeunes majeurs, celles-ci ont été de plus en plus marquées par la contractualisation. Il est important de rappeler que la contractualisation est un processus complexe (ce qui implique une certaine conception de l'État et aussi du social) qui passe à travers l'objet du contrat, mais ne se limite pas à celui-ci. La contractualisation mélange un ensemble de dispositifs dont la vocation est l'insertion et, donc, elle est la partie visible de l'arrivée de nouvelles politiques sociales fondées notamment sur des récits individuels, sur la relation individuelle et sur la mise en perspective par le projet (Guimard, Petit-Gats, 2010).

En ce qui concerne le CJM, pour comprendre les modes de fonctionnement de cette technologie de gouvernement, il faut reprendre certains aspects du Contrat jeune majeur. Il sera important de comprendre le contexte dans lequel le contrat émerge, les changements dans les politiques publiques de protection qui ont bouleversé ses objectifs initiaux et aussi ses dynamiques et logiques actuelles. Le Contrat jeune majeur émerge en France, dans un contexte marqué par une série de transformations (d'ordre législatif principalement) dans les politiques de protection de l'enfance. La première, en raison de la loi n 74-631 du 5 juillet 1974, se réfère à l'abaissement de l'âge de la majorité civile (de 21 à 18 ans), qui a produit un revirement sur les façons de prendre en charge les enfants protégés par le dispositif de protection à l'enfance. À cause de l'abaissement de l'âge et, par conséquent, du " vide juridique " créé par cela, il semble important que le gouvernement atténue les effets indésirables de cette loi (Capelier, 2015).

Ainsi, en suivant cette perspective de l'atténuation des effets du rabaissement d'âge, deux décrets sont créés, en 1975, pour instituer une protection administrative et une autre judiciaire s'adressant aux jeunes âgés de 18 à 21 ans. La promulgation de ces deux décrets conduit à la création d'une nouvelle catégorie juridique : le jeune majeur ${ }^{5}$. Les jeunes majeurs sont des jeunes âgés entre 18 et 21 ans qui ont des difficultés d'intégration sociale par manque de ressources ou de soutien familial adéquat. Pour faire face aux effets de la réduction de l'âge de la majorité et aux lacunes qu'elle a laissées en ce qui concerne la protection et l'assistance aux jeunes de plus de 18 ans, les sujets eux-mêmes ont alors la possibilité de demander la poursuite d'une mesure pour les protéger après la majorité.

Afin de comprendre ce contexte, il est important d'expliquer brièvement le fonctionnement du dispositif français de protection, dont la particularité est précisément le fait qu'il repose sur une dualité, qui a été accentuée en 1983 à cause des lois de décentralisation. Autrement dit, le dispositif est formé par deux versants : l'un administratif relevant du Département (Aide sociale à l'enfance - ASE), et l'autre judiciaire au sein de l'État (Protection judiciaire de la jeunesse - PJJ). Les deux, jusqu'en 2007, étaient chargés de fournir un soutien matériel, éducatif et psychologique aux enfants et à leurs familles lorsqu'ils sont confrontés à des difficultés qui mettent en danger leur santé, la sécurité, léducation. Cela signifie que les jeunes de 18 à 21 ans pouvaient bénéficier, jusqu'à ce moment-là, d'une protection de ces deux institutions, en obtenant un statut administratif ou juridique de jeune majeur. Sur le plan judiciaire, ces sujets pouvaient demander une Action de protection des jeunes majeurs (APJM) et, dans l'administratif, il y avait la possibilité du Contrat jeune majeur (Jung, 2010).

\footnotetext{
4 Depuis 1792, la majorité en France était fixée à 21 ans.

5 Il est important de souligner que cette catégorie ne comprend pas seulement les jeunes qui, pendant l'enfance et l'adolescence, ont été placés, mais tous les jeunes qui se trouvent en situation de difficultés d'insertion.
} 
Cependant, en raison de nombreux changements dans le système de protection français, notamment en raison de la Réforme de la protection de l'enfance en 2007 , on assiste à un désengagement de l'État par rapport aux procédures civiles et au soutien éducatif offert jusqu'à ce jour aux jeunes majeurs par PJJ, qui est devenu responsable seulement pour l'action pénale. Face à un tel scénario, les départements sont confrontés à une demande accrue de protection aux jeunes majeurs, puisque la demande de protection judiciaire devient subsidiaire et justifiée, seulement en cas d'échec de la mesure administrative (Jung, 2010).

La durée du contrat peut varier d'un mois à un an et il peut être renouvelé jusqu'à 21 ans. Pour la signature du contrat, il est nécessaire que les jeunes répondent à une série de " rites administratifs ». Le premier consiste à l'acheminement de la demande au Secteur éducatif auprès des jeunes majeurs (SEJM). Dès la réception de la demande, un entretien est immédiatement fixé. Même avant l'entretien, il est préférable que le jeune, avec l'aide de son éducateur, envoie au SEJM un dossier dont le principal document est le formulaire de demande d'Accueil provisoire jeune majeur (APJM). Parmi les documents justificatifs du formulaire il y a la lettre adressée à l'inspecteur de l'ASE, où le jeune doit justifier sa demande et présenter son projet éducatif et professionnel.

Le deuxième rite comprend « l'entretien dans le SEJM » au cours duquel on attend la participation active des jeunes ; cela signifie produire un récit de tout leur parcours dans le cadre de la protection sociale française. À partir de ce moment, le dossier est transmis par la Commission SEJM à la Commission jeune majeur, qui émettra un avis définitif sur l'acceptation de la demande et la durée du Contrat jeune majeur.

Le troisième rite se réfère à la " signature du contrat ", quand aura lieu le dernier entretien avec le jeune et, par conséquent, l'évaluation finale par l'inspecteur de l'ASE. La signature du contrat marque formellement l'accès des jeunes à un autre statut - jeune majeur - qui vient avec l'annonce de la fin de certains privilèges de la minorité, en particulier la fin de l'obligation d'assistance. En raison de la création de la catégorie jeune majeur, apparaît en France un nouveau " marché de services " spécialisés, comme la Suite éducative, avec les modes de fonctionnement spécifiques tant éducatif que matériel (Guimard, Petit-Gats, 2011).

Ainsi, cette proposition soulève aussi le défi de réfléchir sur les limites des politiques de protection, en particulier sur la possibilité de reconnaissance de ce que peut le sujet, à savoir, sa puissance de mettre en place de nouveaux modes d'existence. Et sur ce point-là, je partage l'intérêt de Schuch (2013) sur les surprises que nous réservent les sujets sur le plan de leurs complexités, et comment ils peuvent apporter de nouvelles complexités au scénario des politiques de protection, en particulier celles liées aux jeunes majeurs. Compte tenu de la proposition de penser les sujets en matière de leurs complexités, je présente, ci-dessous, des extraits ethnographiques des expériences de cinqjeunes de la Suite éducative (Auguste, Albert, Sophie, Alice et Victor).

\section{Auguste : des excuses sur un morceau de papier}

Auguste est guinéen et quand j'ai fait sa connaissance, son Contrat jeune majeur était presque terminé. Ce fut un moment très délicat pour le jeune parce qu'il avait manqué la date limite de demande de renouvèlement du CJM et il avait besoin le plus vite possible de remplir le formulaire de demande du CJM et de rédiger sa lettre de motivation. En outre, la situation de ses papiers restait irrégulière. Lors d'un rendez-vous avec sa référente, il était avec sa petite amie. Les deux semblaient avoir une relation marquée par la complicité, elle soutenait le développement de son projet. Tandis qu'ils parlaient, la jeune fille tenait tout le temps la main du jeune. Et, parfois, il penchait sa tête vers ses genoux, montrant sans honte sa faiblesse à ce moment-là. Il a très mal réussi à l'école, l'année a été perdue pour lui. Ce qui était une préoccupation majeure en ce qui concerne

$6 \quad$ Pour une question d'éthique, les prénoms des jeunes ont été changés. 
la demande de renouvèlement du CJM. Mais, il a dit que son stage allait très bien et qu'il s'était appliqué, parce qu'il aimait beaucoup le travail. La référente a souligné que ce point était à remarquer dans sa demande, à savoir : même s'il avait des problèmes à l'école, le stage se passait très bien.

Tout au long de la conversation, nous avons parlé du CJM et de sa logique de fonctionnement, en particulier, des exigences imposées aux jeunes. J’ai demandéà la référente si le contrat imposait des contraintes aux jeunes. Elle a dit que ce n'était pas seulement cela. Par exemple, a-t-elle dit, je suis dans ma maison, avec ma famille, et je dois tout laisser pour travailler. Ceci est une contrainte. " Maintenant, pour ces jeunes, en particulier ceux qui ne sont pas français, le contrat est leur vie même, c'est bien plus qu'une contrainte. Parce que tu sais que si tu n'as pas de papiers, si tu n'as pas un contrat, tu ne peux pas rester en France. " Pendant qu'elle parlait, je regardais le jeune, qui restait la tête basse, visiblement angoissé par rapport à sa situation qui était exactement celle énoncée par la référente.

Quelques jours après ce rendez-vous, j’ai eu une conversation avec la référente sur Auguste. Elle était très inquiète de la situation et, surtout, elle se faisait du souci pour lui par rapport à l'évaluation du jeune par l'ASE. Elle était préoccupée parce que le renouvèlement du contrat du jeune était en jeu. Il fallait être très prudent sur la manière d'écrire (quoi écrire et avec quels mots). Elle m'a dit qu'elle n'a eu aucun contact avec lui pendant plusieurs jours et qu'il n'a pas donné de retour sur le formulaire et la lettre de motivation. Il avait manqué la date limite. Elle m’a montré l'ébauche de la lettre qu'il avait écrite. En fait, c'était un morceau d'une feuille de cahier, écrit des deux côtés. D’un côté, avec l'écriture de sa petite amie, les points qu'il devait développer (son projet pour ses prochains mois); de l'autre, il y avait le récit du jeune sur sa situation. Dans la lettre, le jeune parlait de ses échecs, de son impuissance face au projet qu'il avait promis à ses parents et aussi à l'ASE. Il a commencé en disant que, au début, il pensait qu'il serait en mesure de gérer son autonomie, de vivre seul et de conduire son projet. Mais il s'était trompé, il n'était pas capable de faire les choses tout seul. Il ne pourrait pas tenir la promesse qu'il avait faite à ses parents : avoir une formation, un emploi et la citoyenneté française. Il ne pourrait pas rentrer à la maison et dire à ses parents qu'il avait réussi. Ce projet serait accompli par son frère cadet qui est venu avec lui en France. Ce morceau de papier contenait également des excuses.

\section{Albert : « Je suis un artiste en herbe »}

Le jeune, dont les parents sont marocains, est né en France. Il avait 14 ans quand il est arrivé à la Suite et son parcours à l'ASE est long et a commencé depuis l'enfance, quand il avait 7 ans. Il est passé par différents foyers et familles d'accueil. Les derniers mois ont été particulièrement difficiles pour lui. Le départ de la Suite est proche et le jeune n'a pas obtenu son baccalauréat (bac) à la fin de l'année dernière. En général, quand un jeune n'a pas eu le bac, il peut le repasser l'année suivante, s'il a obtenu la moyenne pour cela. Mais cette situation n'est pas la réalité de la plupart des jeunes en situation de Contrat jeune majeur. Si le jeune a déjà 20 ans ou qu'il a presque cet âge, il n'aura pas la chance de repasser le bac. Il est prêt à quitter la Suite éducative, il a besoin de quelque chose qui lui permette une insertion plus immédiate et un logement. La réalité des jeunes en situation de Contrat jeune majeur est soumise à la contrainte du peu de temps pour l'insertion et pour faire une formation. Le contrat est une des raisons pour lesquelles la plupart des jeunes choisissent une formation plus courte et plus technique. En matière d'insertion, un baccalauréat professionnel (bac pro) ou baccalauréat technologique (bac techno) est plus facile et produit des résultats plus immédiats qu'un baccalauréat général. Les études longues ne sont pas encouragées, parce que le temps passe très vite et, dans la meilleure des hypothèses, ces jeunes ont trois ans pour s'insérer. 
Donc, sans la possibilité de repasser le bac, Albert veut tenter le Bafa'. Lors d'une de nos conversations, il avait l'air à la fois motivé et triste. Motivé pour toutes ces possibilités qui semblaient s'ouvrir devant ses yeux (à cause de son départ), et triste parce qu'il fallait quitter la Suite. "C'est bizarre, partir de la Suite après si longtemps ». C'est bizarre, étrange et triste, parce que c'est une partie de lui et aussi de son histoire qui restent là. Il ne semblait pas préparé, il semblait même en détresse face à l'effort pour faire croire que tout allait s'arranger en quelque sorte. À sa faveur, il avait la citoyenneté française. Parmi ses désirs, celui d'être un artiste : faire du théâtre, jouer de la guitare, réciter de la poésie. Mais pourquoi n’avait-il pas fait ce choix avant ? Curieusement, le bac choisi n'avait rien d'artistique, ce n'était pas un bac en sciences humaines, mais en sciences exactes. Il avait choisi un bac dans le domaine des machines industrielles. Le choix du jeune a été motivé par le conseil de son père. "C'est mon père qui m'a dit qu'il y avait beaucoup de boulot dans ce domaine ". Ce serait un projet de ton père ? Ai-je demandé au jeune pour comprendre pourquoi il n'avait pas fait la formation dans le théâtre. «Ce que j’ai fait là... les trois ans, j’ai fait n’importe quoi, j’aurais dû pas prendre la formation que j'ai fait. Parce que là j'ai perdu trois ans. J’ai raté mon bac et là j’ai arrêté trois mois, quoi. Mais en même temps j'ai un diplôme, j’ai un BEP. Mais après, de toute façon je reprendrai mes études. Là je suis jeune, j’ai quel âge ? J'ai 20 ans ». Mais au-delà du conseil de son père, le jeune a dit qu'il ne connaissait pas avant sa " capacité pour le théâtre ». «Et là tout le monde dit que je joue bien, que j’ai un potentiel. Que je crois en moi, quoi ! J'ai un potentiel. Même un des éducateurs m’a dit que je suis un artiste en herbe. C'est un artiste qui va devenir un grand artiste plus tard. Là, je suis là. Un artiste au niveau est là. Il faut que je monte les échelons. C'est comme un peu un ascenseur, il monte les échelons! Il monte les étages ! Mais moi, il faut que je monte tous les échelons !»

Il y aurait parmi ces décisions, ou plutôt ces choix, le poids de ce qui est espéré par l'ASE, par les politiques de protection françaises ? À ce moment-là, il était difficile de différencier ce qui était le désir de ce qui était le projet, ce qu'il pourrait être (sur le plan de ses volontés et de ses compétences) ou ce qu'il devrait être (dans la logique d'une insertion et de l'autonomie). Parallèlement au Bafa, Albert a également pour but d'obtenir la Garantie jeunes ${ }^{8}$, mais cela ne sera possible qu à partir du moment où il devra quitter la Suite. Mais tous ces projets feraient l'objet d'une réunion avec toute l'équipe des éducateurs de la Suite, le mardi, jour des réunions de synthèse. Ce jour-là, la fin de la réunion serait consacrée à la préparation du départ du jeune. Cette rencontre a également pu compter sur la participation de la représentante du Théâtre du Fil, où le jeune veut faire son stage du Bafa.

Préparer la sortie des jeunes, qu'est-ce que cela signifiait pour Albert ? Qu'est-ce que cela signifiait pour les éducateurs? Comment prépare-t-on ce moment ? La chef de service a pris la parole en premier. Elle a dit qu'Albert avait beaucoup de projets, mais qu'ils dépendaient de ceci ou de cela. Autrement dit, il y avait beaucoup d'idées, mais concrètement elle voulait savoir comment ces idées fonctionnent et si elles seront possibles. Le jeune avait trois idées en tête : faire le Bafa, être hébergé au Théâtre du Fil et demander la Garantie jeunes.

L'impression que j'ai eue est que tous avaient de grandes expectatives (ou même des certitudes) sur les bonnes nouvelles qu'ils imaginaient que la représentante du Théâtre du Fil leur apporterait et que le devenir du jeune serait assuré à partir de cette conversation. Cependant, quand elle a pris la parole, les expressions, de tous ceux qui ont écouté attentivement, ont commencé à changer. Le théâtre traversait des problèmes structurels et ne pouvait pas héberger Albert en ce moment. Ce qui était certain c'est que le jeune ferait son stage d'animateur au théâtre. Mais il n'aurait pas un endroit où vivre à partir de janvier 2017. Ceci, si le CJM était renouvelé le 30 septembre 2016. Cependant, le jeune semblait sûr que le contrat serait renouvelé jusqu’à janvier. Un des éducateurs lui a demandé ce qu'il aurait l’intention de faire si le renouvèlement du contrat était rejeté.

\footnotetext{
Brevet d'aptitude aux fonctions d'animateur.

8 La Garantie jeune est un dispositif créé par le gouvernement pour donner aux jeunes (de 16 à 25 ans) « la chance d'une intégration sociale et professionnelle grâce à un parcours intensif de formation et d'accès à l'emploi ». Disponible sur Internet : http://www.gouvernement.fr/action/la-garantie-jeunes.
} 
Albert semblait être très confiant. En fait, dans son discours, cette possibilité semblait impensable, car il avait tout expliqué, il a écrit toute sa vie dans la lettre de motivation. Léducateur a insisté sur le fait que la question ne devait pas le troubler, mais il fallait se prévenir : « Ils peuvent te demander pourquoi tu vas demander de l'aide pour faire un Bafa, s'ils t’avaient déjà financé auparavant. Tu dois avoir un plan B " (les éducateurs parlent beaucoup du plan B à la Suite).

Dans la salle, le climat est devenu progressivement plus « lourd », tous semblaient partager la difficulté que ce moment leur imposait : ils avaient besoin de trouver une sortie. En même temps, c'est comme si tous aussi attendaient quelque chose d'Albert, ce qu'il aurait à dire pendant le temps qu'il lui restait avant son départ. Dans ce climat, les questions ont donné lieu à un grand silence, tout le monde regardait le jeune, en attendant un mot, une réaction. Il n’avait rien à dire, mais il a su « casser » cette atmosphère lourde : « Pourquoi tout le monde me regarde ?» Dit-il en souriant. "Plus précisément, qu'est-ce qui se passera ?», demande le stagiaire. Ce fut certainement la question que tout le monde se posait à ce moment-là, mais ils ont préféré la contourner, parce qu'il n'y avait pas de réponse. Il n'y avait rien de concret. En l'absence de réponses, la chef de service a demandé au jeune s'il avait des doutes ou s'il voulait dire ou demander quelque chose. Quelque chose qu'ils pouvaient encore faire pour lui, sauf décider où il habiterait après son départ de la Suite. Le jeune a dit non : " aucun doute et aucune demande à faire " (ou plutôt, c'était déjà fait). Puis, la chef de service a demandé : « tu peux expliquer à tout le monde le projet que tu présenteras à l'ASE ? ". Albert ne voyait aucune nécessité de faire ce que proposait la chef de service. Mais elle a insisté sur le fait qu'il était important que tout le monde connaisse le projet qu'il allait proposer.

Visiblement contrarié, le jeune a expliqué que son projet se composait de trois points principaux : obtenir le Bafa, faire le stage au Théâtre du Fil et demander la Garantie jeunes quand il sortirait de la Suite. Dans l'impossibilité d'être logé par le Théatre, Albert a dit qu'en fin de compte, il allait vivre pendant un certain temps avec sa mère, qui vit seule. Mais, en même temps qu'il voyait la possibilité de vivre avec sa mère, il savait que cela ne serait pas facile. Sa mère le dérange beaucoup ; pour que l'on se fasse une idée, il a expliqué, « elle pense que je suis dans une famille d'accueil. Si elle sait que je suis ici, elle va m’embêter !». « Tu veux qu'elle ne sache rien de ta vie, mais tu veux vivre avec elle ?!», a demandé le stagiaire en souriant. Si c'était vraiment possible de vivre avec sa mère, je me demande pourquoi il aurait fait un si long parcours à la Protection de l'Enfance. La chef de service a rappelé que si le jeune avait l'intention de vivre avec sa mère, elle devrait le savoir avant. Il devait savoir si cela était effectivement une possibilité. « Tu as besoin d'anticiper les choses !», a soutenu la chef de service.

Après cette réunion, j'ai rencontré Albert d'autres fois, l'une d'elles a été dans son studio. Ce jour-là, je lui ai posé des questions sur ses sentiments vis-à-vis de son départ de la Suite éducative. "Je vis au jour d'aujourd’hui, pas l'avenir ", a déclaré le jeune.

\section{Sophie : « toujours dire la vérité » n'est pas une bonne stratégie}

Sophie est de la Guinée Conakry et est arrivée en France quand elle avait 12 ans. Son départ, m’a-t-elle expliqué, a été motivé par des problèmes de santé et par la possibilité d'avoir la formation tant désirée dans le domaine du Droit. Quand elle était petite, elle a découvert qu'elle avait du diabète, à un niveau très grave. Aujourd’hui encore, elle est suivie médicalement et elle suit un traitement avec de l'insuline injectable. À Conakry, elle vivait avec sa mère et son grand-père. Ses parents se sont séparés, sa mère est guinéenne et son père sénégalais. Elle n'a pas vu son père depuis l'enfance, mais garde avec affection sa photo dans son portable. Elle aspire à le rencontrer à nouveau et à vivre au Sénégal quand elle aura terminé sa formation. 
Elle fait un DUT ${ }^{9}$ en Droit à Paris 13. Elle avait commencé une licence, mais elle n’a pas pu continuer, c'était très difficile pour elle. Elle est travailleuse et bien dévouée aux études. Elle dit qu'elle ne veut pas du tout travailler comme avocate, ce qui l'intéresse c'est la carrière diplomatique, donc après le cours elle veut suivre une spécialisation en Relations internationales. Le désir de suivre une telle carrière l'accompagne depuis longtemps et elle a persisté, malgré les discours décourageants de l'éducatrice du foyer où elle a été placée avant d'aller à la Suite. L'éducatrice a mis en doute le potentiel de la jeune et a insisté sur le fait que le CJM vise aux formations de courte durée et qu'il n'est pas pour les études supérieures. Aujourd'hui, Sophie est parmi les quelques jeunes de la Suite éducative qui font des études supérieures.

Comme pour les autres jeunes de la Suite, la demande de renouvèlement de son titre de séjour (Vie privée et familiale) a été refusée ; j’ai appris cette nouvelle lors d'un rendez-vous de la jeune avec sa référente. Ce jour-là, quand la jeune est entrée dans la salle, je pouvais voir que quelque chose lui était arrivée. Elle ne semblait pas contente et conviviale comme d'habitude. Elle était très inquiète et triste. La préfecture de police avait rejeté sa demande de renouvèlement du titre de séjour, affirmant que la jeune femme était toujours en contact avec sa famille et qu'elle n'avait pas de liens durables sur le sol français (elle n'était pas mariée à un français et elle n’avait pas d'enfants, donc, elle n'avait pas de famille ici). Ce n'était pas une bonne stratégie « toujours dire la vérité ", expliquait la jeune. La référente se sentait très coupable de ce qui était arrivé, «je ne suis pas juriste, cela n'est pas mon métier. Je me sens coupable de l'avoir guidée à écrire ce qu'elle ne devait pas écrire. C'est Sophie qui va souffrir les conséquences ", se lamentait-t-elle.

Dans sa demande, la jeune n'aurait pas dû mentionner qu'elle avait des contacts avec sa mère et son grand-père maternel, étant donné que l’une des conditions pour ce genre de titre est justement de ne pas avoir de contact avec la famille. À partir de la date de réception de la lettre de la préfecture de police, la jeune avait deux mois pour faire un recours. La référente commence à recueillir la documentation (les bulletins de l'école, diplôme d'obtention du bac général, passeport, acte de naissance, certificat médical, etc.). Autrement dit, il y a tout un processus de préparation d'un ensemble cohérent de documents qui sont en conformité avec le dispositif juridique. La référente pense que la question du diabète peut être un bon argument, mais peut-être qu'il ne suffira pas. Elle demande à Sophie de prendre contact avec son médecin afin qu'il puisse certifier de son problème de santé et qu'il parle de l'importance de suivre son traitement en France. L'éducatrice a également suggéré à Sophie que son petit ami, qui a la nationalité française et qui travaille dans l'armée, fasse une lettre en disant qu'ils sont ensemble en France. La jeune était très bouleversée et angoissée par les nouvelles. Elle avait peur de devoir retourner dans son pays, sans pouvoir poursuivre ses études et sans commencer la carrière souhaitée. Elle a beaucoup pleuré. En plus de la préoccupation avec le titre de séjour il y en avait une autre : l'attente de renouvèlement du Contrat jeune majeur. Le renouvèlement du CJM a également été un élément pour composer le dossier de demande de recours auprès de la préfecture de police.

Lorsque le jour du rendez-vous à l'ASE est arrivé, afin de renouveler son contrat, Sophie avait déjà déposé auprès de la préfecture de police une demande de titre de séjour étudiant. Même si ce titre est plus précaire, en ce qui concerne la possibilité de travail, il permet à la jeune de régulariser sa situation en France. Dans l'une des salles du nouveau Service jeune majeur, le renouvèlement du CJM de la jeune serait décidé. Le coordinateur du service a commencé son discours en reprenant les points du projet de la jeune, lesquels justifieraient la demande. Ensuite, il a commencé à poser une série de questions qui sont suivies par des orientations qui pourraient aider à la jeune, à la fin du contrat. Il a rappelé que le contrat ne couvre pas toute la période de la licence et il lui a demandé si elle avait envisagé un logement, des bourses, etc. La jeune, qui était toujours très éloquente, a pris à ce moment un ton de voix plus bas et a semblé mesurer chaque mot de son discours. La tête baissée, elle dit qu'elle avait pensé demander une bourse d'études et aussi une place dans la maison

Diplôme universitaire de technologie 
universitaire. Il a insisté sur le fait que ce n'étaient que des hypothèses, mais que c'était important d'envisager des alternatives au cas où les choses ne se passent pas bien. Il a demandé si la jeune avait un logement ou quelqu'un sur qui elle pouvait compter en France. Elle a dit qu'elle pouvait vivre avec la famille de son petit ami. Le coordinateur a souligné qu'il était important, si cela arrivait, qu'elle puisse maintenir le rapport avec l'aide sociale. En cela, le coordinateur lui a expliqué que l'ASE a des services qui peuvent l'aider par rapport à la demande de bourse (pour des périodes plus longues).

Pendant l'entretien, la chef de service a demandé l'autorisation d'intervenir et de dire que la situation administrative de la jeune l'empêchait de suivre le projet proposé au CJM. Elle a expliqué que le titre de séjour VPF de la jeune avait été refusé et que cela l'empêchait de travailler. Le coordinateur a demandé si Sophie avait déjà un avocat pour la guider. Elle a dit qu'elle avait déjà demandé un titre de séjour étudiant. Il a demandé si elle savait que c'est un titre plus précaire que le VPF et qu'elle ne pourrait pas travailler normalement. Elle dit qu'elle le savait et qu'elle avait pensé faire de l'alternance (travail/étude) au cours de la deuxième année du DUT. Après avoir expliqué et posé des questions à la jeune, le coordinateur a dit que le contrat serait renouvelé jusqu'à février 2018. Cela signifiait qu'elle pouvait finir le DUT et même commencer la licence. Les renouvèlements de plus d'un an sont également des exceptions.

Quand nous nous sommes retrouvées seules, j'ai demandé à Sophie si elle avait peur que le contrat ne puisse être renouvelé. Elle a dit qu'elle ne le craignait pas, parce que comme elle avait accompli tout ce qui était inclus dans son projet, il n'y avait pas de raisons pour le non-renouvèlement. Sa préoccupation était plutôt la durée qu'ils pouvaient lui accorder.

\section{Alice : « Je ne peux pas faire la volonté de l'État »}

Alice est une jeune congolaise qui a quitté son pays en raison de la guerre civile. Elle a 18 ans et deux filles: l'une a six ans et vit au Congo avec la grand-mère d'Alice, l'autre, de cinq ans, vit avec la mère d'Alice en dehors de Paris. Selon sa référente, la mère de la jeune est en France en situation d'asile. Dans un premier moment, la mère d'Alice a laissé tous ses enfants au Congo. Après, avec le temps, elle a amené les enfants pour vivre avec elle. Alice a eu sa première fille quand elle avait 11 ans. Les deux filles sont le résultat de viols vécus par la jeune dans son pays (il est important de rappeler qu'au Congo le viol est devenu une arme de guerre). Elle est restée très peu de temps à l'école, elle a dit que son pays est très pauvre et que pour avoir accès à quelque chose (y compris la possibilité d'étudier) les personnes doivent appartenir au groupe politique qui gouverne le pays.

Selon les récits de sa référente, la jeune vient à la Suite régulièrement pour les rendez-vous hebdomadaires et elle entretient une bonne relation avec tout le monde. Le problème, c'est que la jeune n'habite pas dans sa chambre. Face à cette impasse, son éducatrice a marqué un rendez-vous avec la chef de service et la jeune pour essayer de comprendre ce qui se passait et aussi chercher des moyens pour résoudre cette situation. Elle ne dort jamais dans sa chambre, qu'elle trouve très petite et pas propre, en particulier les toilettes et la douche qui sont collectifs. L'éducatrice et la chef de service essaient de trouver une solution au problème, car étant en situation de CJM, Alice ne pourrait pas rester dans la maison familiale ou dans l'appartement de son ami, où elle a passé ses nuits. " Où veux-tu rester ?», lui demande la chef de service. Elle répond, la tête baissée et la voix très timide, qu'elle sait que l'appartement de son ami n'est pas sa place et en même temps, elle sait qu'elle ne peut pas rester dans la maison de sa mère.

La chef de service fait référence à la possibilité qu' a la jeune de pouvoir rester dans un Foyer jeune travailleur (FJT), comme le foyer où habite son amie. La référente lui rappelle que le problème de rester dans un FJT est que la jeune n'aura pas le soutien éducatif, ce qui est très important pour elle maintenant, surtout compte tenu de tout le travail que la référente et son éducatrice à l' ASE font pour qu'elle puisse rester progressivement avec sa fille. La chef de service s'impatiente et argumente : "Elle a signé un contrat pour être ici ". 
À cela, la jeune répond, la tête toujours baissée et avec la voix timide : " Je ne peux pas faire la volonté de l'État ". La référente rappelle également qu'en FJT elle ne pourra pas recevoir sa fille. " Je veux aller dans un foyer normal ", dit la jeune en essayant de trouver un moyen de sortir de l'impasse. L'éducatrice lui explique qu'à son âge, il n'y a pas de foyer normal. « Maintenant, c'est terminé, il n'y a que des endroits comme la Suite ou le FJT ». Après, l'éducatrice rappelle que la jeune termine sa formation en juin et que son projet inclut sa fille. Mais il n'y a pas d'argument qui puisse convaincre la jeune, elle reste irréductible. Ce n'est pas un problème de vivre seule, le problème est que la jeune ne veut pas du tout vivre dans l'appartement qu'elle considère comme sale. La référente dit : " Tu ne peux pas rester dans cette situation ». "C'est dommage de laisser la Suite juste pour une chambre ", ajoute la chef de service ne voyant pas d'alternative à cette impasse imposée par la jeune. La professionnelle quitte la salle sans trouver de solution. Elle dit simplement à la jeune : tu dois réfléchir à ce que tu veux vraiment ». Lorsque la chef de service quitte la salle, nous trois (Alice, l'éducatrice et moi) essayons de trouver une alternative. La référente semble se motiver un peu avec les possibilités, mais pas la jeune. Je lui demande si le problème serait de rester loin de sa fille. Alice dit non, que sa fille était très bien avec sa grandmère et que c'était mieux pour elle comme ça. Et elle insiste, encore, sur le manque d'hygiène des toilettes. Elle raconte qu'elle a une énorme cicatrice de la césarienne de sa fille et qu'elle a peur d'être infectée. «Je commence à me gratter chaque fois que j'utilise les toilettes ». Elle me raconte la situation difficile qui est arrivée lors de la naissance de son premier enfant, expliquant le problème de la cicatrice. Elle a beaucoup souffert parce que sa fille pesait $4 \mathrm{~kg}$ à la naissance. Elle dit que les césariennes sont terribles au Congo sans péridurale, avec seulement une simple anesthésie. Elle pensait qu'elle allait mourir, qu'elle ne saurait pas supporter tout cela.

Dans la période de vacances d'été, Alice est partie de la Suite éducative. Elle a été transférée dans un autre service jeune majeur en dehors de Paris, « juste à cause d'une chambre ». Elle avait fait un choix. Le problème de la chambre, en particulier l'hygiène, n'a pas été inclus dans les négociations. C'était comme si à chaque fois qu'elle utilisait les toilettes, elle devait faire face aux situations de violence qu'elle avait vécues dans son pays.

\section{Victor : « Je suis venu pour le bien de quelqu'un »}

Victor est de la Côte d'Ivoire. Le jeune vient d'une famille pauvre, sa mère a connu beaucoup de difficultés pour élever ses trois enfants (Victor et ses deux sœurs cadettes). En Côte d'Ivoire, on lui a promis une vie meilleure en France, avec la possibilité de trouver un emploi :

Ce n'est pas moi qui ai pris la décision. Je suis venu pour le bien de quelqu'un. Il m’a fait de fausses promesses. Tu vois ? Tu viens en France, là-bas c'est bien, une belle ville. Tu vas aider ta mère au bled, parce que je sais que là-bas est chaud. [...] Ma mère a cotisé, elle a réuni une somme pour mon départ. [...] C’est cher, 1.100 euros comme ça, je pense. [...] deux semaines après mon arrivée, il a dit au revoir !

Victor est arrivé en France avec le passeport d'une autre personne, avec l'aide d'un " passeur ". Il a vécu quatre mois de peur, dans un pays inconnu, même s’il parle la même langue. " Je galérais au début ». Le jeune n’a eu aucun contact avec sa mère pendant quatre semaines. Enfin il a parlé avec sa mère et il a pu lui expliquer un peu tout ce qui se passait; elle a beaucoup pleuré. Mais il lui a dit qu'il resterait pour voir ce qui allait se passer. Être courageux à cette période-là ce n'était pas une question de choix, c'était une obligation.

Comme Sophie, Victor a également fait face à des problèmes pour obtenir le titre de séjour (VPF). Sa dernière demande a été refusée et la raison était la performance du jeune homme à l'école avant de se rendre à la Suite éducative et aussi le fait qu'il a eu des contacts avec sa famille. Mais contrairement à Sophie, Victor est assez pragmatique sur la possibilité de retourner dans son pays, s'il n’obtient pas ses papiers : 
Si jamais la Préfecture refuse de me donner le titre, je reste ici pour quoi ? C'est comme si tout ce que je faisais était vraiment un gros n'importe quoi. Je vais rester là pourquoi ? Je vais attendre que la meuf va me donner mes papiers ? Qui va me donner mes papiers ? Personne. Je ne peux rien faire. Je ne peux pas travailler, je ne peux rien faire. Je ne sais pas. Je reste chez moi, c'est mieux. Et puis, rester ici ? Attends, c'est vrai ! Pourquoi tu restes là, tu cherches quoi ? Tu n’arrives pas, tu ne peux pas travailler. Tu vas voler ? Tu vas faire quoi ? Tu fais quoi ? Rien. C'est mort ! Rentre chez toi.

En ce moment, le jeune fait un bac Électrique et prévoit de commencer en 2017 un BTS. En 2018, son contrat se termine. Cependant, ce n'est pas exactement cette formation qu'il voulait faire en premier. Son projet était de faire mécanique ferroviaire. C'est à partir des conseils de son professeur, à l'époque, que le jeune a fini par changer de choix :

Parce que quand je suis passé au test, et ma professeure qui allait me guider dans ma vie m’a dit : 'Je peux te trouver ce que tu veux, mais je préfère que tu partes pour l'électricité, parce qu’à lélectricité, tu peux travailler dans divers domaines.

Toutefois, s'il obtient ses documents, le jeune n'a pas l'intention de retourner dans son pays. Dans ses récits, on peut voir comment il se sent reconnaissant pour tout ce que la France a fait pour lui jusqu'à présent. Il reconnaît que les premiers mois ont été très difficiles et pleins d'incertitudes, mais il a été accueilli et a eu l'occasion de commencer sa formation. Victor est déjà depuis trois ans en France ; même s'il a galéré au début, il n’a pas renoncé. Il avait une mission et il a trouvé un soutien pour l'accomplir. « Je suis resté et ils se sont vraiment occupés de moi ". S’il part, son sentiment sera d'avoir pu accomplir beaucoup de choses. Mais s'il reste en France, il a l'intention de se dédier encore plus, étant donné que tout a un temps et qu'il faut vivre des expériences. " Je reste, je travaille. Et puis on verra. Je suis là, je pense ici ».

Par rapport à son pays, sa famille est la chose qui lui manque le plus. La possibilité d'aider sa mère fait qu'il reste en France. Il envoie, chaque mois, une partie de son argent en Côte d'Ivoire, pour aider sa mère et ses sœurs. « Chaque fin de mois, je tente d'envoyer quelque chose à elle pour se défendre. C'est ma mère ! Grâce à elle que je suis ici ». Il dit qu'il ne pense pas vraiment au moment de son départ de la Suite, parce qu'il préfère s'occuper du moment présent et de toutes les possibilités que cela offre. « Ce n'est pas vraiment le départ. Moi, je pense d'abord au présent. Je ne peux pas faire cela. Étape par étape. On va doucement. Pour l’instant, je suis à la Suite. Et je suis là pour quelque chose ».

\section{Modes de se construire comme sujet : migration, désir et temporalités}

À partir des cinq expériences rapportées, je voudrais maintenant soulever quelques questions. La première, qui me semble essentielle pour comprendre la façon dont les jeunes peuvent se construire en tant que sujet dans cette technologie de gouvernement fait référence à la migration. Parmi ces cinq jeunes, quatre ont quitté leur pays, encore mineurs, à la recherche de meilleures conditions de vie en France. Ces jeunes, quand ils arrivent en France, dans de nombreux cas, ils n'ont même pas de passeport et migrent avec l'aide d'un " passeur ", comme ce fut le cas de Victor. Il n'est pas rare que les familles réunissent des économies pour payer une personne qui leur promet non seulement l'entrée en France, mais, surtout, une vie meilleure. L'arrivée dans le pays de destination est accompagnée par l'entrée de ces mineurs dans le Dispositif de protection à l'enfance français. Alors, quand ils sont mineurs, la question des papiers (ou plus précisément leur absence) ne pose pas de problème. Mais la situation change complètement lors de l'arrivée à la majorité. À partir de ce moment, les démarches pour obtenir les papiers ont tendance à se complexifier et à imposer des limites à ce que ces jeunes voulaient être. L'obtention d'un emploi est soumise à la régularisation de leur situation sur le 
sol français. Comme cela apparaît dans les récits de Victor, l'impossibilité d’obtenir le titre de séjour, en fin de compte, invalide tous les efforts faits pour avoir une formation et un travail. "C'est comme si tout ce que je fais était vraiment un gros n'importe quoi ".

Selon le récit de l'une des éducatrices de la Suite, la situation des jeunes sans-papiers s'aggrave de plus en plus. À la Suite éducative, ces derniers temps, ils ont eu 19 cas de jeunes sans-papiers. Cette constatation appuie la demande des éducateurs afin que l'Association puisse avoir un avocat pour les aider dans les procédures qui ne sont pas de la responsabilité du travail éducatif. Et contrairement à ce que l'on pourrait imaginer, le départ du pays de provenance est un mouvement difficile pour beaucoup de jeunes. Comme l'a soutenu une autre éducatrice, la plupart quittent la maison des parents, quand ils sont encore mineurs, sans savoir où ils vont. Les jeunes voyagent vers d'autres pays où ils ne connaissent personne et parfois ils ne parlent même pas la langue locale. Et le point le plus intéressant de la réflexion qu'a fait l'éducatrice est la question du choix. Qui a choisi ou qui a voulu partir ? Et pourquoi voulaient-ils partir ? La plupart ne veulent pas partir, ce qui apparaît très clairement dans le récit de Victor. "Ce ne fut pas moi qui ai pris la décision. Je suis venu pour le bien de quelqu'un. On m'a fait de fausses promesses ". Parfois, ce sont les parents qui prennent la décision de faire partir les enfants : « Les parents ont fait ce choix pour sauver les enfants ». Et les raisons qui les font partir sont nombreuses : la situation de guerre, la précarité, une vie meilleure et la possibilité d'aider la famille, les conflits familiaux, etc.

Il y a des jeunes qui partent en raison d'une " mission " donnée par les parents et/ou par la famille ou d'une mission qu'ils se donnent à eux-mêmes (Etiemble, 2008). Cette idée de la mission apparaît dans le cas de Victor qui a quitté la Côte d'Ivoire pour faire une formation et pour travailler, mais surtout, pour aider sa famille, de sorte que chaque mois il se soucie d'envoyer quelque chose pour aider sa mère et ses deux sœurs. On peut aussi l'observer dans l'expérience d'Auguste quand il raconte son impuissance par rapport au projet qu'il avait assumé vis-à-vis de ses parents. Il ne pouvait pas revenir en arrière et dire à ses parents qu'il avait échoué. Maintenant, ce projet serait réalisé par son frère.

Les mineurs mandatés ont pour mission (ou se donnent pour mission, 's'auto-mandatent') de se rendre en Europe pour travailler et faire vivre la famille restée au pays. Cette mission est parfois associée à un projet d'études et d'acquisition d'un métier socialement valorisé [...]. Eux sont davantage inscrits dans une logique d'ascension sociale (Etiemble, 2008, p.180).

La réflexion que je fais, en ce moment, c'est qu'il ne s'agit pas seulement d'un projet individuel, comme prévu par les modes de fonctionnement du CJM. Il est souvent un projet familial. La migration « mobilise toute la famille, qui est stratégiquement organisée pour envoyer certains membres et garder les autres à la maison " (Lobo, 2006, p.113) (c'est moi qui traduit). Il est important de noter que la notion de famille est utilisée dans son sens le plus large. Décider lequel des enfants va émigrer n'est pas un choix aléatoire. Une telle décision " doit être en conformité avec les intérêts et les besoins du groupe familiale » (Lobo, 2006, p.113) (c'est moi qui traduit). À la limite, on peut dire qu'un autre contrat a été préalablement établi avec la famille. Il y a aussi une dette assumée avec la famille.

Il faut souligner que le projet d'immigration n'est jamais exclusivement individuel, en fait, il est le résultat d'une "connexion du sujet avec la société d'origine" (Jardim, 2015, p.66) (c'est moi qui traduit). C'est ainsi que le "projet migratoire" ne prend forme qu’à partir d'une série de décisions qui sont traversées par des projets nationaux, par des connexions historiques (coloniales) entre les lieux d'origine et de destination, ainsi que par des médiations étatiques qui interviennent dans les calculs individuels et collectifs. C'est par un complexe tissage avec tous ces éléments que la définition du comment et du pourquoi de l'immigration devient une option possible pour les sujets (Jardim, 2015, pp.66-67) (c'est moi qui traduit). C'est dans ce sens que le projet individuel 
accordé dans le CJM peut être mieux compris. De telle sorte que les perspectives de succès ou d'insuccès d'un tel projet participent au tissage du projet migratoire et sont soumises à l'exercice de la réflexivité des sujets (Sayad, 1998 ; Jardim, 2015).

Et ce projet ne coïncide pas nécessairement avec l'idée d'étudier et d'obtenir une formation, comme dans le cas de Sophie. À la base de ce projet, le but peut être simplement d'obtenir les papiers pour travailler. Il implique d'avoir une vie meilleure en France, mais aussi de fournir les conditions pour la survie de la famille, de ceux qui sont restés. Ainsi, le projet, que l'on veut individuel, mais qui parfois est familial, est non seulement une obligation pour certains jeunes, mais aussi la vie en soi. Donc, on pourrait penser que l'une des façons de se construire comme sujet est liée à cette " circulation " non seulement entre les pays, mais entre les différentes cultures, et aux limites imposées à la vie quotidienne de ces jeunes à cause d'une négociation entre un projet individuel (à remplir pour correspondre à ce qui est exigé par les logiques de contractualisation), et un projet qui est familial.

Il est important de prendre en considération que ce processus permet de se détacher des signatures identitaires et même de les dépasser. Sophie, qui a des origines ${ }^{10}$ guinéennes et sénégalaises, a appris à sélectionner dans quelles circonstances, et toujours ainsi de façon contingente, chacune de ses origines lui permet une meilleure performance. Donc, quand elle pense à faire une carrière diplomatique, c'est plus productif de se construire en tant que Sénégalaise, mais quand il s'agit de parler de l'éducation qu'elle a reçue, elle parle en tant que Guinéenne. Mais elle laisse en deuxième plan ces deux origines, quand elle dit qu'elle est totalement adaptée à la vie parisienne. Il y a également une négociation qui est faite en termes de relations de parenté établies entre ces jeunes. Et ils apprennent ça, à partir des démarches de demande de titre de séjour. Ce n'est pas une bonne stratégie de " toujours dire la vérité", a appris Sophie. Qu'est-ce que cela signifie ? Cela veut dire qu'il faut réinventer les relations de parenté, les liens qu'ils établissent, la force de ces derniers et la possibilité de les refuser si nécessaire. Ce sujet qui se construit à partir de nombreuses relations parentales, devient un sujet qui peut être " isolé » en territoire étranger, ou qui n’a personne sur qui compter dans les moments difficiles : il est complètement seul, il n’a même pas des nouvelles de sa famille. Donc, ce sont des façons de se construire comme sujet qui ne sont pas stables et n'acquièrent aucune forme définie. Elles sont temporaires et sont tout le temps en cours de renégociation. "Ce sont des mouvements d'émancipation dont le sens politique s'élabore contre et/ou à l'écart de l'ordre qui institue leur identité » (Agier, 2013).

Il est important d'observer la manière dont ces jeunes font et vivent leur vie, malgré et par rapport aux limites qui sont imposées dans leur vie quotidienne et comment ils trouvent des sorties et des lignes de fuite. «Comment ils font face à ces forces dans leurs projets de vie et découvrent les potentiels pour le changement, même s'ils sont petits" (Biehl, 2016, p.418) (c'est moi qui traduit). La proposition est de se déplacer par rapport à une conception réductrice de l'expérience de ces jeunes qui est liée à ce qui est attendu en matière de performance par les politiques publiques de protection. Et l'un des chemins se trouve dans la façon de décrire la capacité créatrice des sujets, leur potentiel d'agence. Rappelons que "l'agence ", ici, est pris non seulement " comme résistance » ou " résultat » d'un processus qui, dans le cas de ces jeunes, serait le développement de leur projet individuel (devenir autonome), ou même la réalisation d'une action. " [...] Il est nécessaire de regarder les capacités d'agence des personnes, comment les personnes se mobilisent, pourquoi et comment elles imaginent et souhaitent " (Biehl, 2016, p.419) (c'est moi qui traduit). Et pour cela, il faut faire attention à la fois aux négociations avec les réalités et les limites posées par celles-ci, ainsi que les refus de s'engager avec elles.

10 Il faut souligner les différentes variables qui permettent de définir les « origines » de ces jeunes. Au-delà des documents (comme le passeport, la carte d'identité ou l'acte de naissance) qui attestent officiellement une origine géographique, un âge, etc., les jeunes définissent leurs origines à partir d'éléments très éloignés des considérations administratives. «Ils décrivent ainsi les contours d'un territoire à taille plus humaine. Chacun est, de par ses expériences de vie - et non de par son extranéité -, porteur d'une origine hybride articulée autour de plusieurs lieux ». (Przybyl, 2016) 
À partir des expériences des jeunes, on peut trouver des pistes sur les manières dont chacun négocie avec des réalités posées par cette technologie de gouvernement. « Parfois il faut savoir comment jouer le jeu du contrat ", explique une des éducatrices. Sophie négocie comme quelqu'un qui fait des études supérieures, elle est une bonne élève, dédiée, et rêve de faire une carrière diplomatique. On peut observer à partir de sa performance lors du rendez-vous pour le renouvèlement du contrat : voix plus basse, gestes plus contrôlés. Elle a répondu à toutes les questions qui lui ont été posées. Elle était déjà informée de ses possibilités. Elle a appris à s'approprier la technologie de gouvernement. Elle a bien appris à jouer «le jeu du contrat ». À la limite, elle a constaté aussi que « toujours dire la vérité » n'est pas une bonne stratégie. Et le coordinateur a réalisé qu'il valait la peine d'investir dans la jeune et il lui a expliqué toutes les autres possibilités d'aide que l'ASE pourrait offrir. Auguste négocie aussi, mais à partir d'une autre place qui n'est pas celle d'une personne qui a réussi à l'école ou au travail. Comme il n'a pas de points forts à souligner, comme l'a fait Marie Hélène qui négocie à partir de son potentiel, il a recours à la description des difficultés qu'il a vécues, sa souffrance et l'apparente incapacité de réaction. C'est sa petite amie qui écrit les points qu'il doit développer dans la lettre. Il se construit comme " victime » et transfert à son frère cadet la tâche de réaliser le projet familial. Il faut observer cet autre lieu à partir d'où le jeune négocie, car il souligne également un moyen de tester les limites de cette technologie de gouvernement. Et cette façon de se construire comme un sujet peut également être observée dans le cas d'Albert qui, compte tenu de l'impossibilité d'être hébergé par le Théâtre du Fil, lance au groupe d'éducateurs la possibilité de vivre avec sa mère, ce qui n’a jamais été viable tout au long de son parcours de placement. Une autre place possible est celle qui refuse l'engagement avec ces réalités. Tel est le cas d'Alice qui préfère quitter la Suite éducative et surtout le suivi éducatif qui a été fait pour qu'elle puisse vivre avec sa fille. Elle se construit comme sujet, soit quand elle nie l'accueil à la Suite éducative, soit quand elle rompt avec la violence qu'elle a vécue dans son pays. Elle " ne pouvait pas faire le désir de l'État ", même si elle a signé un contrat et une dette, comme le lui rappelle la chef de service.

Le Contrat jeune majeur pourrait donc être pensé comme une technologie de gouvernement qui est formée par deux types de segmentarité. Une « segmentarité molaire », qui est l'ordre des règles, des bureaucraties et des connaissances établies, très évidentes dans la conception du projet et des rites administratifs pour la signature du contrat. Et une " segmentarité moléculaire " qui correspond à une série de pratiques qui fonctionnent d'une autre manière, à partir de différents agencements, tels que les appropriations que les jeunes font des discours des politiques et des éducateurs et aussi comme bifurquant du projet formalisé dans la lettre afin de le concilier avec ce qu'ils veulent faire. De telles pratiques sont de l'ordre du désir (Deleuze, Guattari, 1996).

En observant les expériences des jeunes, on peut remarquer la présence d'une dialectique permanente (et sans synthèse) entre le projet (sur le plan de ce qui est attendu pour obtenir un CJM) et le désir, ce qui échappe aux engrenages mêmes de la technologie du gouvernement. Parfois, le projet et le désir coïncident, comme dans le cas de Sophie, elle a proposé un projet qu'elle a toujours désiré : avoir une formation dans le Droit et faire une carrière diplomatique. La possibilité de retourner dans son pays à cause de problèmes avec les papiers constitue une menace à son projet, mais surtout à tout ce qu'elle a imaginé et souhaité pour sa vie. Parfois, le projet bifurque (comme une manière de négocier avec la réalité et ses limites). Dans un premier moment, il arrête de correspondre à ce qui était de l'ordre du désir. Mais à la fin, il trouve la motivation pour réconcilier le projet et le désir. Et c'est peut-être même, à ce moment-là, l'occasion de découvrir ce que les jeunes savent et ce qu'ils aiment faire, comme ce fut le cas de Victor. Et il avait d'ailleurs un potentiel reconnu par les éducateurs à l'école, qui lui ont recommandé le bac et non pas un CAP.

Une troisième possibilité peut être observée dans l'expérience d'Albert. Dans son cas, le projet et le désir ne sont pas en accord dès le début. Il ne fait pas ce qu'il désirait faire, mais ce qui pourrait correspondre à ce que l'on attend en matière de politiques de protection et aussi en termes familiaux. Une façon de s'adapter à ce qui lui est demandé et, donc, aux modes de fonctionnement de cette technologie de gouvernement. « Le cours 
de théâtre n'est pas considéré favorablement par l'ASE. Ce genre de chose est difficile de passer à l'ASE ", se souvient la référente du jeune. Ainsi, c'est seulement à la fin de son CJM qu'Albert décide de reprendre ce qui relevait de l'ordre du désir. Et à ce moment-là, il est de nouveau capturé par la segmentarité molaire. Le théâtre ne pouvait pas le recevoir, il devait trouver un emploi pour être en mesure de se maintenir et de trouver un logement. Rappelant également que ce que chaque jeune désire et/ou imagine pour sa vie est parfois traversé par les désirs des autres. Ces désirs se confondent. Albert a fait du désir de son père son désir.

Il est important de remarquer que cette relation entre le projet et le désir, ou entre " segmentarité molaire » et " segmentarité moléculaire » n'est pas stable non plus. Au contraire, l'incitation entre les deux est permanente. Donc, contrairement à ce qu'on pourrait penser, la « segmentarité molaire » n'empêche pas l'émergence des pratiques moléculaires, au contraire, elle les encourage. Ainsi, comme la segmentarité molaire stimule l'émergence de la segmentarité moléculaire, l'inverse est également possible, à tel point que, en fait, l'une ne peut exister sans l'autre. Plus l'organisation molaire est forte, plus elle finit par susciter une molécularisation de ses éléments. Cela signifie qu'il n'est pas suffisant de définir la bureaucratie tout simplement à partir d'une " segmentarité dure ", car il y a, en même temps, un ensemble de " perversion bureaucratique " et une " inventivité ou créativité permanente qui s'exerce contre les règlements créatifs " (Deleuze, Guattari, 1996). À la suite de cette perspective, on peut dire que le Contrat jeune majeur cherche à la fois à produire des cadres, établissant des règles, des moralités, les savoirs et les pratiques bureaucratiques, et, en parallèle, il attise un ensemble de pratiques créatives des sujets (les jeunes et aussi les professionnels qui les accompagnent) qui sont de véritables « lignes de fuite».

La question de la temporalité est un autre point à souligner par rapport aux modes par lesquels les jeunes se constituent en tant que sujets. Beaucoup a été dit sur la durée du CJM, en particulier, parce qu'elle impose un temps très court pour que les jeunes deviennent autonomes et ainsi qu' ils conquièrent leur insertion sociale. Cependant, ce que j'ai pu observer, à partir des expériences des jeunes de la Suite éducative, c'est qu'il ne s'agit pas d'une préoccupation pour la durée du contrat, mais pour une question beaucoup plus complexe qui est liée à la façon même dont les jeunes conçoivent le temps. Ils ont une autre façon de comprendre et de vivre le temps qui se concentre sur le présent (les évènements) et ils n'ont pas l'ambition de contrôler leur devenir (ce qui est l'objectif principal de $\mathrm{CJM}$ ). Nous sommes confrontés à d'autres temporalités qui se situent au-delà de l'idée courante que les jeunes vivent le moment présent et qu'ils ne se préoccupent pas de leur avenir. Il ne s'agit pas d'un simple "présentéisme ", mais d'une conception du temps qui considère que chaque évènement peut transformer complètement leurs expériences. On peut observer qu'il n'y a pas une préoccupation à contrôler le temps (ce qui serait une préoccupation centrale de la technologie de gouvernement). Car c'est précisément là, dans cette ouverture à des temporalités différentes qui se croisent, que se trouvent la créativité, la puissance et le désir. Alors, quand Albert dit : «Je vis au jour d’aujourd'hui, pas l'avenir », ou lorsque Victor explique : « Moi, je pense d'abord au présent. [...] On va doucement ", on peut penser que les jeunes signalent cette ouverture à " l'inattendu » et donc au " devenir ", dans le sens philosophique. Il s'agit d'accepter les changements et de ne pas essayer de les contrôler. Vivre chaque évènement en reconnaissant le potentiel de transformation qu'il porte. Comme l'a dit un des jeunes de la Suite : "Je ne m' inquiète pas pour le moment du départ de la Suite. Il n'y a pas comment savoir ce qui vient après, tout peut changer. Je ne sais pas ce qui m'attend ». Le devenir « [...] contient donc en germe tout ce qui n'existe pas encore, tout ce qui est proprement impensable et impossible " (Mozère, 2005).

En ce sens, il est intéressant de penser que même quand il y a un manque de mouvement et apparemment pas de sorties, on peut penser au devenir (Biehl, 2016, p.418) (c'est moi qui traduit). La réunion, dans laquelle l'équipe d'éducateurs prépare le départ d'Albert de la Suite, ou le rendez-vous d'Auguste avec sa référente, ou encore le rendez-vous d'Alice avec sa référente et la chef de service, les trois situations montrent ce manque de réaction aux évènements de la part des jeunes et des éducateurs. Cependant, l'ouverture au devenir permet 
d'entrevoir comment les choses seraient autrement. Il ne faut pas penser à ce que ces jeunes vont devenir en raison des échecs sur le plan d'un parcours destiné à être envisagé par le CJM. Quand la professeure a dit à Sophie que les études supérieures ne sont pas pour elle, et qu'elle devait comprendre que dans le cadre du CJM, les jeunes font des études de courte durée, elle délimitait, à priori, ce que la jeune pourrait devenir. La professeure entrevoyait la fin du parcours, le produit de ce que la contractualisation devrait produire. Et, ce faisant, elle a fini par ignorer tout le mouvement à entreprendre par la jeune (avec toutes ses déviations et imprévisibilités) pour poursuivre d'autres possibilités. Et aujourd'hui, la jeune fait des études supérieures pour suivre une carrière diplomatique. Bien sûr, la perspective du devenir est évidemment tensionnée par la dimension pragmatique des logiques du contrat : trouver un emploi, avoir un logement, obtenir une formation, etc. Et le contrat impose aux jeunes le poids des attentes qui ont été déposées sur eux, tel que cela apparaît dans la réflexion que fait la chef de service face à l'indécision d'Alice « Mais tu as signé un contrat pour être ici ". Après un investissement fait en termes éducatifs et financiers (il y a une dette). Donc, devenir un acteur de théâtre, par exemple, ne constitue pas un « bon " projet : c'est instable et ne permet pas la maîtrise du devenir. Cependant, le devenir permet de penser comment les choses pourraient se passer différemment, il considère ce qui est de l'ordre du désir. Le devenir permet de réfléchir sur ce que ces jeunes peuvent en tant que sujet : Un devenir artiste, par exemple, un « artiste en herbe ».

Le devenir est ouvert à l'inattendu et au changement. Même quand il y a un manque de mouvement, lorsque les circonstances sont intraitables, le devenir répond à la manière dont les choses pourraient être autrement. Il y a aussi une autre temporalité présente là et, qui sait, le devenir la contemple. Alors, un potentiel reste ouvert à de nouveaux points de départ, de nouveaux types d'imagination, de nouveaux désirs et de nouvelles négociations (Biehl, 2016, p.418) (c'est moi qui traduit).

Ainsi, considérer cette manière dont les jeunes vivent le temps permet une ouverture aux nouveaux points de départ et aux négociations avec les limites imposées par les réalités. Et cela passe par une remise en cause des questions que le CJM impose à ces jeunes, en particulier, celles qui sont liées au devenir (Où vont-ils arriver ? Qu'est-ce qu'ils deviendront ?). La proposition est de déplacer ces questions, de façon à ne pas concentrer les efforts sur les issues de ces vies, mais sur ce qui se passe dans le « milieu » où l'on « atteint la plus grande vitesse. Ce milieu est précisément là où les temps les plus différents se communiquent et se croisent dans un tourbillon " (Pelbart, 2010). C'est précisément dans le milieu que se trouve le devenir, la puissance : ce que peut un sujet.

Ainsi, tout au long de l'article, je cherche à souligner l'importance de la proposition d'une Anthropologie du Sujet (qui prendra en compte le devenir) pour réfléchir sur les expériences des jeunes par rapport au Contrat jeune majeur, en tant que technologie de gouvernement. Lorsque l'on considère les modes par lesquels les jeunes peuvent se constituer comme sujet et les devenirs qui sont impliqués dans ce processus, je ne prétends pas que les politiques de protection puissent répondre aux spécificités et aux demandes de chaque sujet. En effet, toute politique, précisément à cause de son caractère public, suit certaines catégories d'homogénéisation (tels que la vulnérabilité, la situation de violation des droits, etc.). Mais en même temps, cette proposition permet de mettre en perspective cette conception d'un « universel » qui peut recouvrir une totalité sur le plan de sa diversité. Le défi est donc l'exercice d'identifier et de réfléchir sur les limites des politiques, notamment en ce qui concerne la reconnaissance d'agence des sujets. Ceci est un exercice permanent de réflexivité sur les possibilités de concevoir et de mettre en œuvre des politiques de protection plus plurielles, qui ne possèdent initialement aucun problème. 
Date de soumission: le 05 novembre 2018

Date d'acceptation: le 06 mai 2019

Révisé par: Bertrand Bogo

\section{Bibliographie}

AGIER, Michel. 2013. La condition Cosmopolite. L'anthropologie à l'épreuve du piège identitaire. Paris : La Découverte.

BIEHL, João. 2016. “Antropologia entre o inesperado e o inacabado”. Horizontes Antropológicos, 46: 389-423.

CAPELIER, Flore. 2015. « Penser la transversalité dans l'accompagnement des jeunes majeurs ». Permettre la Jeunesse - Vie Sociale, 12: 55-69.

CRUZ, Fernanda. 2014. Jovens em devir: invenção de novas possibilidades de vida para além da institucionalização. Tese de doutorado, Florianópolis, Universidade Federal de Santa Catarina.

DELEUZE, Gilles. 1989. " Un concept philosophique ». Cahier Confrontation, 20: 89-90.

.; GUATTARI, Félix. 1996. Mil Platôs. Capitalismo e Esquizofrenia, v. 3. São Paulo: Editora 34, s/p.

Disponible: 〈http://ghiraldelli.pro.br/wp-content/uploads/Gilles-Deleuze-Mil-Plat\%C $3 \%$ B4s-Vol.-3.pdf> Date d'accès: 17 juin 2019.

. 1997. “Devir-Intenso, Devir-Animal, Devir Imperceptível”. In: Mil Platôs: Capitalismo e Esquizofrenia. vol. 4. Trad. Suelly Rolnik. São Paulo: Ed. 34 : 11-113.

ETIEMBLE, Angelina. 2008. «Parcours migratoires des mineurs isolés étrangers, catégorisation et traitement social de leur situation en France ». E-migrinter, 2: 180-185.

FASSIN, Didier ; MEMMI Dominique (dir.). 2004. Le gouvernement des corps. Nouvelle édition [en ligne]. Paris : Éditions de l'École des Hautes Études en Sciences Sociales. Disponible sur Internet : <http://books. openedition.org/editionsehess/1478>. ISBN : 9782713225710>. Date d'accès: 17 juin 2019.

FOUCAULT, Michel. 2008. Segurança, Território, População: curso dado no Collège de France (1977-1978). Trad.

Eduardo Brandão. São Paulo: Martins Fontes.

. 1979. Microfísica do poder. $25^{\mathrm{a}}$ ed. Trad./Org.: Roberto Machado. Rio de Janeiro: Edições Graal. . 2010. A Hermenêutica do Sujeito. Trad.: Marcio Alves da Fonseca; Salma Tannus Muchal. $3^{\underline{a}}$ ed. São Paulo: Editora WMF Martins Fontes.

. 2012. "A Ética do Cuidado de Si como Prática da Liberdade". In: Ditos e Escritos, v. 5: Ética,

Sexualidade, Política. Tradução de Elisa Monteiro e Inês Autran Dourado Barbosa. $3^{\underline{a}}$ ed. Rio de Janeiro: Forense Universitária: 264-287.

GUIMARD, Nathalie ; PETIT-GATS, Juliette. 2010. «Écrits de jeunes en quête de statut ». Recherches Familiales, $7:$ :115-125.

. 2011. Le Contrat Jeune Majeur : Un temps négocié. Paris : L'Harmattan.

JARDIM, Denise. 2015. "O projeto migratório palestino: uma releitura de narrativas e conflitos intrafamiliares em uma perspectiva etnográfica”. História Oral, 18(1): 63-92. Disponible sur Internet: https://www.lume.ufrgs.br/bitstream/handle/10183/141906/ooo989328.pdf ?sequence=1 Date d'accès: 17 juin 2019.

JUNG, Céline. 2010. L'Aide Sociale à L'Enfance et les jeunes majeurs : comment concilier protection et pratique contractuelle? Paris : L'Harmattan.

LOBO, Andréa de Souza. 2006. Tão longe, tão perto: Organização familiar e emigração feminina na Ilha de Boa Vista, Cabo Verde. Tese de doutorado em Antropologia Social, Universidade de Brasília -UNB, Brasília. 
MOZÈRE, Liane. 2005. « Devenir-femme chez Deleuze et Guattari. Quelques éléments de présentation ». Cahiers du Genre, $38: 43-62$.

PELBART, Peter Pál. 2010. O tempo não-reconciliado: Imagens do tempo em Deleuze. São Paulo: Perspectiva.

PRZYBYL, Sarah. 2016. Territoires de la migration, territoires de la protection. Parcours et expériences des mineurs isolés étrangers accueillis en France. Géographie. Université de Poitiers (France). Disponible sur Internet : https:/|tel.archives-ouvertes.fr/tel-01429926. Date d'accès: 30 juin 2019.

SAYAD, Abdelmalek. 1998. A Imigração ou os Paradoxos da Alteridade. São Paulo: Editora da Universidade de São Paulo.

SCHUCH, Patrice. 2013. Práticas de Governo, Cultura e Subjetividade: etnografia dos circuitos de atenção à “juventude violenta”. Projeto aprovado no edital universal do CNPq (Mimeo). .; RIBEIRO, Fernanda; FONSECA, Claudia (orgs). 2013. "Apresentação: Infâncias e crianças: saberes, tecnologias e práticas". Civitas, 1: 205-220.

STRATHERN, Marilyn. 2006. O Gênero da Dádiva: Problemas com as mulheres e problemas com a sociedade melanésia. Trad. André Villalobos. Campinas-SP: Editora da Unicamp.

\section{Fernanda Rifiotis}

Post-doctorante Université Fédérale de Rio Grande do Sul,

Département de deuxième et troisième cycle en Anthropologie sociale, Porto Alegre/RS, Brésil http://orcid.org/oooo-0002-7307-2254

E-mail: frifiotis@gmail.com 